\title{
Estudios de audiencias y medios comunitarios: apuntes para un reencuentro necesario*
}

\author{
Susana M. Morales* \\ Recibido: 2019-06-3 • Enviado a pares: 2019-06-28 \\ Aprobado por pares: 2019-08-02 - Aceptado: 2019-09-20 \\ https://doi.org/10.22395/angr.v18n36a4
}

\begin{abstract}
Resumen
Este artículo presenta aprendizajes y debates sobre la relación entre conocimiento académico y proyectos de comunicación no lucrativos, vinculados a los resultados de un proceso de investigación sobre audiencias con cuatro radios comunitarias de gestión cooperativa del Gran Córdoba (Área Metropolitana de Córdoba). Esa investigación tuvo como objetivo contribuir a la sostenibilidad de las radios comunitarias a través de la producción de conocimiento sistemático sobre sus audiencias reales y potenciales. Se realizaron ocho estudios, dos para cada radio, uno de carácter cualitativo y uno de carácter cuantitativo. Los cuantitativos consistieron en encuestas poblacionales sobre consumos mediáticos, especialmente orientadas a la radio y a medios locales, así como a niveles de satisfacción con la información local y regional. Los cualitativos se desarrollaron con entrevistas en profundidad y grupos focales, según cada caso, y abordaron modalidades de escucha de radios, distintos modos de identificación con las radios en cuestión, así como cuestiones relacionadas con la experiencia de vivir en las ciudades en las que se sitúan las radios.
\end{abstract}

Entre los hallazgos más importantes, aparece un conjunto de datos que permiten problematizar la noción de consumo convergente, ya que es posible reconocer modalidades de consumo audiovisual que articulan medios analógicos y digitales, en el que se articulan vínculos de larga data con determinados medios, con fuertes componentes afectivos y de costumbre con nuevos tipos de consumo. Tanto el proceso de investigación como sus resultados posibilitaron reconocimientos que fortalecieron a los medios en cuestión, pero también actualizaron un modo de relación entre investigación y medios comunitarios en el que se articularon saberes académicos, inquietudes generadas en la práctica y abordajes teórico-metodológicos en los que se produjeron aprendizajes donde los resultados excedieron el alcance esperado de la investigación.

Palabras clave: estudio de audiencias; radio; medios comunitarios; sociología de la comunicación; impacto de la comunicación.

Artículo producto del proyecto de investigación Radios cooperativas y públicos. Estudios de consumos y audiencias en el Gran Córdoba. Parte I y II. El proyecto, dirigido por la doctora María Liliana Córdoba, contó con el asesoramiento de la profesora María Cristina Mata y lo llevaron a cabo integrantes del programa de Estudios sobre Comunicación y Ciudadanía del Centro de Estudios Avanzados de la Facultad de Ciencias Sociales de la Universidad Nacional de Córdoba, Argentina.

* Doctora en Ciencias de la Información, Universidad de la Laguna, España. Docente investigadora del Área de Estudios de la Comunicación del Centro de Estudios Avanzados de la Facultad de Ciencias Sociales, Universidad Nacional de Córdoba, Argentina. Correo electrónico: susanamoralesar@gmail.com. Orcid: https://orcid.org/0000-0003-0491-1920 


\title{
Audiences and Community Media Studies: Notes for a Necessary Reencounter
}

\begin{abstract}
This article presents lessons learned and debates about the relationship between academic knowledge and nonprofit communication projects, linked to the results of a hearing research process with four community radios of cooperative management in Greater Córdoba (Córdoba Metropolitan Area). This research aimed to contribute to its sustainability through the production of systematic knowledge about its real and potential audiences. Eight studies were conducted, two for each radio, one of a qualitative nature and one of a quantitative nature. The quantitative one consisted of population surveys on media consumption. especially oriented to radio and local media, as well as to levels of satisfaction with local and regional information. The qualitative ones were developed through in-depth interviews and focus groups, according to each case, and addressed radio listening modalities, different ways of identifying with the radios in question, as well as issues related to the experience of living in the cities where the radios were located.

Among the most important findings, there is a set of data that makes it possible to problematize the notion of convergent consumption, since it is possible to recognize forms of audiovisual consumption that articulate analog and digital media, in which long-term links are articulated with certain media, with strong affective and custom components with new types of consumption. Both the research process and its results enabled recognition that strengthened the media in question, but also updated a relationship between research and community media in which academic knowledge, concerns generated in practice and theoretical methodological approaches were articulated in the that there were learnings that exceeded the research results.
\end{abstract}

Keywords: audience study; radio; community media; communication sociology; audience measurement; communication research; communication impact.

\section{Estudos de audiências e meios comunitários: anotações para um reencontro necessário}

\section{Resumo}

Este artigo apresenta aprendizagens e debates sobre a relação entre conhecimento acadêmico e projetos de comunicação não lucrativos, vinculados aos resultados de um processo de pesquisa sobre audiências com quatro rádios comunitárias de gestão cooperativa do Grande Córdoba (área metropolitana de Córdoba, Argentina). Esta pesquisa teve como objetivo contribuir para a sustentabilidade das rádios comunitárias por meio da produção de conhecimento sistemático sobre suas audiências reais e potenciais. Foram realizados oito estudos, dois para cada rádio, um de caráter qualitativo e um de caráter quantitativo. Os quantitativos consistiram em entrevistas com a população sobre o consumo midiático, especialmente orientadas à rádio e aos meios locais, bem como a níveis de satisfação com a informação local e regional. Os qualitativos são desenvolvidos com entrevistas em profundidade e com grupos focais, segundo cada caso, e abordaram modalidades de escuta de rádios, diferentes modos de identificação com as rádios do estudo e questões relacionadas com a experiência de morar nas cidades nas quais se encontram as rádios. Entre os achados mais importantes, aparece um conjunto de dados que permite problematizar a noção de consumo convergente, já que é possível reconhecer modalidades de consumo audiovisual que articulam meios analógicos e digitais, no qual são articulados vínculos de longa data com determinados meios, com fortes componentes afetivos e de costume com novos tipos de consumo. Tanto o processo de pesquisa quanto seus resultados possibilitaram o reconhecimento que fortaleceu os meios em questão e atualizaram o modo de relação entre pesquisa e meios comunitários no qual foram articulados saberes acadêmicos, dúvidas geradas na prática e abordagens teórico-metodológicas em que foram produzidas aprendizagens cujos resultados superaram o avanço esperado da pesquisa.

Palavras chave: estudo de audiências; rádio; meios comunitários; sociologia da comunicação; medição de audiência; pesquisa sobre a comunicação; impacto da comunicação. 


\section{Introducción}

Este artículo tiene como objetivo divulgar un conjunto de debates y aprendizajes de una experiencia de investigación sobre audiencias con cuatro radios comunitarias del Gran Córdoba (Área Metropolitana de Córdoba)', aprendizajes en torno a las potencialidades de articular procesos de investigación con proyectos audiovisuales del sector comunitario que permiten renovar, tanto las preguntas de investigación como los abordajes teórico metodológicos. A su vez, son aprendizajes sobre una relación que habilita a que los colectivos de comunicación puedan poner en tensión sus propias certezas, desarrollar sus intuiciones y obtener información que les permita potenciar sus proyectos. Y finalmente, se producen resultados que permiten avanzar en debates más amplios de los estudios de comunicación, ligados a las modalidades y transformaciones de los consumos audiovisuales y la conformación de audiencias.

Partimos de reconocer que estas radios comunitarias tienen un conocimiento escaso e intuitivo, tanto de sus oyentes como del escenario comunicacional en el que se desarrollan. En parte, porque las tareas de gestión y programación concentran la mayoría de los esfuerzos de los colectivos radiofónicos, pero también por el modo en que esos colectivos consideran a esas audiencias en sus prácticas cotidianas.

La relevancia de la pregunta en torno a las audiencias de las radios comunitarias tiene que ver con las condiciones y posibilidades de sustentabilidad de los proyectos político-comunicacionales en un contexto en el que nuevamente, se tiende a homogeneizar y concentrar el sistema de medios audiovisuales de nuestro país. Además, supone preguntas en torno a la incidencia de estos proyectos en el marco de un proceso de profundas transformaciones en el consumo de medios, entre otras razones, por las transformaciones tecnológicas vinculadas a los procesos de convergencia tecnológica. Pero sobre todo, supone preguntas en torno al modo en que las audiencias y sus derechos comunicacionales son incorporados en los proyectos audiovisuales, más allá de su enunciación genérica.

\section{Contexto de investigación}

Para dar cuenta del valor de este proceso de investigación es necesario reconocer el contexto de debates en el que se inserta, ya que el debate, sanción e implementación de la Ley de Servicios de Comunicación Audiovisual n.o 26.522 (LSCA) en nuestro país, junto con transformaciones tecnológicas, implicaron profundas modificaciones en el sistema de medios. Para el sector comunitario, la sanción de la LSCA implicó la salida de la ilegalidad, pero sobre todo significó su reconocimiento como actor central para la

En el artículo no se van a presentar resultados de investigación más que de modo general para especificar algunas de las afirmaciones que se realizan. Esta decisión tiene que ver con que la información producida en el marco del proyecto es propiedad de las radios comunitarias con las que trabajamos, además de que en muchos casos contienen información sensible para las radios y para las organizaciones involucradas. 
democratización del sistema de medios, para promover la pluralidad y la diversidad en la producción comunicacional y el reconocimiento de los derechos comunicacionales de las audiencias. También implicó que se consolidara este sector y se multiplicaran los medios comunitarios ${ }^{2}$ frente a un panorama histórico caracterizado por la concentración de la propiedad de los medios y su carácter marcadamente orientado al sector comercial, pero también concentrado a nivel de producción de contenidos en Capital Federal y en algunas capitales provinciales. Este nuevo contexto, cuyo horizonte fue la transformación del escenario audiovisual nacional en relación con el reconocimiento de la comunicación como un derecho, no estuvo exento de contradicciones. Los conflictos no se dieron solamente con quienes hasta ese momento habían hegemonizado un modelo de producción audiovisual y concentrado la propiedad de los medios, sino también entre los mismos actores que desde distintos ámbitos y prácticas habían construido y sostenido los ejes del modelo audiovisual promovido por la LSCA.

En particular, en la provincia de Córdoba, durante este periodo se gestó un importante conjunto de radios comunitarias, sobre todo al interior de la provincia ${ }^{3}$. Para todos esos medios, la sanción de la LSCA en 2009 significó un impulso al crecimiento, creación y consolidación de sus proyectos (Monje, Rivero y Zanotti, 2018; Villegas Uribe, 2016; Kejval, 2017). Sin embargo, dado lo reciente del proceso y su falta de consolidación, constituye un sector altamente vulnerable, tanto en términos económicos como por el impacto de las transformaciones en materia de políticas de comunicación orientadas al sector (Segura et al., 2016).

Cuando asumió la presidencia el ingeniero Mauricio Macri en diciembre de 2015, una de sus primeras iniciativas fue modificar la LSCA por decreto, especialmente en los artículos vinculados a la limitación de la concentración de la propiedad de los medios audiovisuales, e intervenir la Autoridad Federal de Servicios de Comunicación Audiovisual (AFSCA), órgano de aplicación de dicha ley ${ }^{4}$ A partir de allí, comenzó una nueva y violenta transformación del panorama audiovisual argentino que profundiza la concentración de la propiedad de los medios y que desanda las búsquedas de pluralidad a través del paulatino vaciamiento de los medios públicos, del cierre de un conjunto de medios y del disciplinamiento de medios no oficialistas a través del manejo de la pauta oficial y de la persecución a sus dueños o gerentes. Este período se caracteriza por la búsqueda de volver a homogeneizar el panorama audiovisual de

En el Informe sobre el relevamiento de servicios de comunicación audiovisual comunitarios, alternativos, populares, cooperativos y de pueblos originarios elaborado por la Red Interuniversitaria de Comunicación Comunitaria, Alternativa y Popular, es posible observar cómo a partir del 2008 -año de presentación, debate y sanción de la LSCA- se produce un crecimiento exponencial de nuevos proyectos de comunicación comunitaria (Riccap, 2019).

3 En el capítulo "Córdoba" del informe de la RICCAP (2019) se puede observar que el 70 \% de los cuarenta y siete medios comunitarios fueron creados a partir de 2008 (Doyle, Meirovich y Morales, 2019).

$4 \quad$ Para dar cuenta de esos cambios, ver Defensoría del Público (s.f.). 
nuestro país y profundizar de manera inédita la concentración de la estructura de propiedad de los medios.

Si bien el proyecto de investigación se elaboró en un contexto previo a este enorme retroceso en materia de democracia comunicacional, su desarrollo se produjo a medida que las radios comenzaban a sentir el impacto de las políticas regresivas: las dificultades para cobrar planes de fomento ya otorgados, la multiplicación exponencial del costo de las tarifas eléctricas y distintos ataques a las redes del sector comunitario. Sumado a ello, hubo un fuerte impacto sobre las condiciones económicas y laborales de los integrantes de los colectivos radiofónicos.

En este marco se desarrolló, en conjunto con cuatro radios comunitarias del Gran Córdoba, un proceso en el que se produjeron distintos tipos de investigaciones en torno a las audiencias reales y potenciales de esas radios, así como sobre los distintos vínculos que esas radios establecen con sus públicos. Se trabajó en dos etapas con cuatro radios comunitarias: Radio Nativa de la ciudad de Unquillo, Radio Inédita de Cosquín, Radio VillaNos de Carlos Paz y Radio Tortuga de Alta Gracia, todas son de creación reciente ya que empezaron a transmitir entre 2008 y 2014. Se realizaron en total ocho estudios, dos para cada radio, uno de tipo cuantitativo y otro cualitativo.

\section{Antecedentes}

El proyecto surge como iniciativa de las emisoras y se enmarcó en una convocatoria específica de la Secretaría de Políticas Universitarias orientada a la economía social -programa de Cooperativismo y Economía Social de la Universidad Nacional de Córdoba-, por lo tanto, solo se trabajó con radios de gestión cooperativa. Si bien los integrantes de las radios con las que trabajamos tienen una preocupación en torno a cuáles son sus audiencias reales, los niveles de conocimiento de las emisoras en sus comunidades, las características a partir de las cuales se reconocen los proyectos radiofónicos, así como las razones para ser o no escuchadas, en general, no cuentan con prácticas sistemáticas que les permitan reconocer sus audiencias y rediscutir o reorientar sus proyectos político comunicacionales o sus estrategias de programación. En buena medida, las urgencias en torno a sostener económicamente los proyectos y a sostener la programación, es lo que tiende a concentrar los mayores esfuerzos, aún más en un momento como el actual en el que los embates de las políticas de comunicación generan una transformación sustantiva de las modalidades de gestión que les dieron origen (Monje y Rivero, 2018; Segura et al., 2018).

En este sentido, se ha de destacar que la cuestión central en el desarrollo de medios comunitarios que produjo investigaciones que generaron desarrollos conceptuales centrales para los estudios de comunicación, quedaba por fuera de lo que estas radios consideraban como parte de la tarea de comunicar. En Desde la experiencia 
al saber. Sistematización de las experiencias de investigación en audiencias (Mata, 2012), se hace énfasis en la importancia que desde la Asociación Latinoamericana de Educación Radiofónica (ALER) se le confirió a la cuestión del conocimiento en distintas áreas, entre ellas, el conocimiento sobre las audiencias. En este sentido, afirma Mata (2012): "Un saber colectivo, enraizado en la práctica y orientado hacia ella. Un saber útil: producto de necesidades y con un horizonte definido, un algo por mejorar, por alcanzar, por superar" (p. 12).

En ese texto que sistematiza investigaciones en torno a las audiencias de las radios educativas y populares, se identificaron desde 1981 veintitrés estudios de distinta envergadura, que se reconocen como un tipo de conocimiento ineludible para diferentes objetivos: la autoevaluación institucional, redefinir o planificar proyectos radiofónicos e institucionales, evaluar proyectos o programas específicos, superar el espontaneísmo y las intuiciones generales vinculados a la experiencia, o simplemente conocer qué sucede con los públicos de las emisoras.

Así, desde las radios se desarrollaron estudios que permitieron conocer a los oyentes, qué se escucha en general, cuáles son los hábitos de consumo y el modo en que se relaciona con el consumo de otros medios; qué se escucha de las emisoras en cuestión, qué se prefiere de ellas; con qué cualidades se las identifica y porqué se las valora; usos informativos y de entretenimiento, expectativas y demandas, entre otras cuestiones.

Una cuestión que destaca en este texto es que los aportes más significativos de estas investigaciones exceden a sus resultados, ya que permiten un conjunto de debates en torno a comportamientos diferenciados de las audiencias de radio, según edades, género, zonas urbanas o rurales, así como a diferentes motivaciones en torno a demandas similares de información. También aportaron al debate sobre la marginalidad de los proyectos comunitarios, lo cual abre las puertas para pensar qué masividad es posible imaginar, diferente a la del mercado; permitieron comprender la centralidad de la radio en contextos locales y comunitarios. Pero sobre todo, aportaron a dar cuenta sobre cómo esos medios son valorados como herramientas de organización, participación y expresión popular, así como sobre su capacidad articuladora de proyectos vinculados a la lucha por la transformación social, en estrecha relación con los vínculos afectivos y emotivos que generan. Asimismo, permitieron reconocer que en términos políticos, una de las dimensiones más relevantes era la capacidad de esas radios para permitir la articulación de sectores populares entre sí, el diálogo y la confrontación entre ellos y otros, y con el Estado.

Esta larga referencia a un texto que pone de relieve el valor que han tenido los estudios de audiencias en proyectos de comunicación comunitaria tiene como objeto destacar hasta qué punto la pregunta por las audiencias ha sido una preocupación 
central de las emisoras para dar cuenta de sus preocupaciones teórico políticas. Además muestra cómo estos desarrollos han sido centrales para problematizar el campo académico y los debates conceptuales en torno a los medios de comunicación.

En nuestro país, algunas radios comunitarias participaron de este proceso ${ }^{5}$. Sin embargo, el periodo de crecimiento y expansión del sector comunitario que inició en el 2009 no contó con un conocimiento sistemático de las audiencias de los medios comunitarios. Existen datos de mercado para las grandes ciudades y proyectos comerciales que sostienen distintas experiencias orientadas a conocer sus audiencias: sus propios estudios cualitativos y cuantitativos, o la información que proveen los análisis de datos de medios digitales.

En términos de conocimiento producido en el marco de políticas públicas, entre 2005 y 2008 se realizó una encuesta de consumos culturales, del Sistema Nacional de Consumos Culturales, y desde el 2013, la encuesta del Sistema de Información Cultural de la Argentina realizó dos encuestas nacionales ${ }^{6}$. Estas encuestas brindan información general, a nivel nacional y desagregada por regiones sobre algunos indicadores que aportan datos estructurales sobre el consumo de medios, entre otros consumos, y permiten leer algunas tendencias generales (Grillo, Papalini, y Benítez Larghi, 2016). Sin embargo, no dan cuenta de realidades locales como las que se abordaron en este proyecto, ni de cuestiones específicas ligadas a distintos usos de los medios. Por el modo en que se publicaron los datos, también es difícil seguir algunas variables ya que se dificulta la comparación. En materia de políticas públicas orientadas al audiovisual, la experiencia del programa Polos Audiovisuales Tecnológicos ${ }^{7}$ desarrolló investigaciones orientadas al desarrollo de estudios sobre consumos y audiencias en distintas ciudades del interior del país (Córdoba y Morales, 2018).

A nivel académico priman abordajes cualitativos sobre recepción y análisis del discurso, dada la dificultad de llevar adelante abordajes cuantitativos por la complejidad organizativa y presupuestaria (Grillo y Segura, 2016). Sin embargo, podemos destacar que en la Universidad Nacional de Río Cuarto se desarrollan estudios locales de manera sistemática hace más de veinte años, dirigidos por la profesora Mabel Grillo. De manera más reciente, estos estudios se desarrollan en algunas universidades del conurbano bonaerense y algunos se realizan en alianza con medios públicos (Monje

Radio Encuentro, en Viedma e Incupo en Chaco.

Los resultados de las encuestas se encuentan disponibles en https://www.sinca.gob.ar/Encuestas.aspx

En el 2011 se creó el programa Polos Audiovisuales Tecnológicos, una de las políticas llevadas adelante por el Consejo Asesor del Sistema Argentino de TV digital abierta, dependiente del Ministerio de Planificación Federal, Inversiones Públicas y Servicios. Este programa estuvo orientado a instalar y fortalecer las capacidades para la producción nacional de contenidos para la TV digital, promovía la igualdad de oportunidades y la disminución de asimetrías entre provincias y regiones para dar cumplimiento al artículo 153 de la Ley 26.522 de Servicios de Comunicación Audiovisual.

$8 \quad$ Los estudios se encuentran disponibles en el siguiente enlace: https://www.unrc.edu.ar/unrc/comunicacion/dptocomunicacion/investigaciones/inv_2.html 
y Mercadal, 2018) y comunitarios (Kejval y Ávila Huidobro, 2017; Segura et al., 2018).

Si bien existen estos datos generales, cuando iniciamos el proyecto solo una de las radios con las que trabajamos contaba con información fiable sobre los consumos de medios en su localidad. Por otro lado, ninguna de las radios contaba con información sobre el mapa comunicacional local -información que tampoco tienen los organismos públicos encargados del desarrollo del Plan Técnico para la regularización del espectroy las distintas propuestas con las que iban a disputar sus audiencias. De ese modo, al formular la investigación también se evidenció que los proyectos comunitarios se insertaron en escenarios radiofónicos de alto nivel de concentración mediática en los cuales, en muchos casos, los medios dominantes son parte de grandes grupos empresariales, tanto locales como nacionales, cuya presencia es muy marcada. Esto da cuenta de las enormes dificultades de reconocer el escenario comunicacional en el que se lanzan las propuestas comunicativas de los proyectos comunitarios.

Aún más, se desconocían las dinámicas de consumo mediático ligadas a escenarios marcados por los procesos de convergencia (Doyle, 2017; Lunt y Livingtone, 2015; Orozco, 2007; Orozco y Miller, 2017). Esto es, de las transformaciones en las prácticas de los oyentes de radio frente al proceso de digitalización en virtud del cual los servicios de comunicación audiovisual que tradicionalmente se ofrecían en soportes analógicos comienzan a estar disponibles en plataformas digitales bajo la organización de nuevos modelos de prestación y negocio (Monje, 2018).

No solo se trataba de una cuestión de acceso a la información y conocimiento sobre los modos en que se configuran las audiencias con quienes las radios comunitarias proponen ponerse en diálogo. De manera complementaria, también operaba entre los integrantes de esas radios un conjunto de nociones sobre los procesos de comunicación y específicamente, sobre el modo en que se piensa a esos sujetos a quienes se pretende interpelar, que dificulta reconocer el carácter histórico y construido de las audiencias, y sobre todo, reconocerlas como sujetos de derecho (Lunt y Livingstone, 2010). En el diálogo con los colectivos radiofónicos fuimos encontrando algunos modos de pensar las audiencias que ya han sido ampliamente discutidos, tanto por los estudios de comunicación como por las redes que articulan proyectos de comunicación comunitarios. Sin embargo, persistían en las prácticas de quienes llevan adelante estos proyectos.

Por un lado, hay una tendencia a confundir gustos, intereses y expectativas propias con las de los públicos a los que quieren llegar. De allí surgen propuestas de programación, narrativas y propuestas informativas y estéticas que se asumen como relevantes para aquellos a quienes se pretende interpelar. De este modo, no hay interrogantes y búsquedas en torno al vínculo que se proponen con sus audiencias. También hallamos nociones empiristas (Mata, 1997), es decir, la idea de que la audiencia preexiste a la 
relación comunicativa que se propone, con demandas y necesidades que solo deben ser conocidas para ser satisfechas. También se identificó la idea de que las audiencias son solo el punto de llegada de sus propuestas, como un simple receptor más o menos activo, con lo cual se cofunde a veces actividad con participación. Se trataba entonces de discutir con quienes llevan adelante la tarea de comunicar y solamente consideran sus intereses, habilidades y gustos, para quienes esa audiencia es poco más que un casillero a completar en un formulario de un plan de fomento.

Por otro lado, existía cierto menosprecio al reconocimiento de las audiencias reales, ya que algunos integrantes de estas radios consideraban que no sería relevante una disputa por la masividad.

En este marco, nuestra investigación buscaba aportar al conocimiento sistemático de las audiencias de esas radios, proporcionar pistas con respecto a posibilidades de crecimiento, así como aportar a la comprensión del modo en que las propuestas radiofónicas compiten y dialogan con otras propuestas mediáticas, para repensar desde ahí las ofertas radiofónicas democráticas y de calidad (Magallanes, Ramos, Castells y Parra, 2010; Kejval, 2017).

\section{Pensar las audiencias}

Para construir estos aportes, fue necesario generar un modo de pensar las audiencias que permita darle sentido a los datos que las radios esperaban. Conocer las audiencias, desde la perspectiva de quienes trabajamos en este proyecto, supone más que un recuento de prácticas de consumo, gustos e intereses (Livingstone, 2005a ; 2019). Conocer las audiencias supone conocer colectivos más o menos unificados en cuanto a gustos, preferencias, necesidades y usos que les otorgan una cierta identidad común. Además, supone reconocer esos hábitos ligados a una marca estructural vinculada a la experiencia cultural de ser público de los medios, reconocerse en sus interpelaciones y constituir identidades colectivas a partir de ese reconocimiento (Mata, 1997). De este modo, conocer las audiencias supone reconocer que han sido modeladas desde la cultura masiva, desde diferentes consumos y prácticas culturales que se articulan con rutinas cotidianas que también están marcadas por las relaciones con esos medios (Mata, 1997).

Entonces, conocer esas audiencias no solo significa saber quiénes miran determinados canales y programas, quiénes escuchan qué radios, sino también por qué y cómo: conocer sus hábitos y preferencias en el marco de una trama más vasta de consumos y prácticas culturales. Es pensar las relaciones de las personas con el medio y la cultura de la que forman parte. Pero también significa conocer las valoraciones sobre el medio y su relación con la vida colectiva, sus demandas y deseos de información y entretenimiento que no están satisfechos por las ofertas existentes. 
Desde este punto de vista, es posible comprender la práctica de comunicar como una interacción con gustos, necesidades, intereses y costumbres de quienes se espera que escuchen los proyectos radiofónicos. Además, podemos pensar esas prácticas de comunicación en el escenario cultural y comunicacional más amplio en el que se insertan al reconocer la complejidad con la que cada propuesta política comunicacional está en diálogo y disputa.

Este conocimiento no supone afirmar esos gustos ni esos intereses, sino reconocerlos para poder entrar en diálogo con ellos. La novedad de las propuestas radiofónicas que aspiran a ser escuchadas no se puede construir por fuera de esa búsqueda por el diálogo con los otros. Reconocer la modelación cultural que fue configurando los hábitos de consumo permite identificar las demandas insatisfechas que existen en la relación con esos medios, las expectativas abiertas y las búsquedas que no están contenidas en las propuestas comunicacionales existentes.

De este modo, al considerar que las radios son espacios de interacción es posible construir propuestas capaces de interpelar y reconstruir el vínculo comunicacional de manera permanente y cotidiana, en busca de alternativas de identificación para que las audiencias se conviertan en públicos de nuestros medios.

\section{Metodología}

A partir de estas premisas comenzó el proceso de investigación con una formulación genérica: estudios cuantitativos y cualitativos para cuatro radios comunitarias de gestión cooperativa del Gran Córdoba de las ciudades de Unquillo, Alta Gracia, Carlos Paz y Cosquín' ${ }^{9}$ esta se fue redefiniendo, en primer lugar, por razones de financiamiento. Por ello, en la primera etapa se realizaron dos estudios cuantitativos poblacionales en las ciudades de Unquillo y Cosquín, y dos de tipo cualitativo en las ciudades de Alta Gracia y Carlos Paz. En la segunda etapa se realizaron estudios cuantitativos allí donde se habían desarrollado los de tipo cualitativos.

Sin embargo, más allá de las características generales del proyecto y de sus resultados, nos interesa rescatar el proceso que se realizó con las radios, que a partir del diálogo con sus integrantes, este fue recuperando sus inquietudes y las fue construyendo como objetos de investigación, generando preguntas específicas y desafíos teórico metodológicos.

Los estudios cuantitativos realizados desarrollaron encuestas poblacionales de carácter residencial y probabilístico, basadas en muestra trietápica, aleatoria,

Estas ciudades se encuentran en un radio de $60 \mathrm{~km}$ de la ciudad de Córdoba. Son ciudades que en los últimos quince años han sufrido enormes cambios demográficos, en los que su población ha crecido de manera exponencial, lo cual transformó su perfil turístico al de ciudades metropolitanas que funcionan en buena medida como ciudades dormitorio para habitantes que desarrollan sus actividades en la ciudad capital. 
con unidades en la primera etapa coincidentes con los límites cartográficos de los radios censales extraídos en primera instancia con probabilidades proporcionales a sus tamaños poblacionales. En la segunda y tercera etapa, con probabilidades inversamente proporcionales a dichos tamaños. La muestra final de trabajo para cada ciudad estuvo compuesta por 400 casos, cantidad que garantiza representatividad para poblaciones de más de 10.000 habitantes $^{10}$, con un error de $+-4,9 \%$ y un nivel de confianza del $95 \%$.

Las dimensiones sobre las que se produjo información a través de las encuestas fueron consumo de medios (televisión, plataformas audiovisuales, diarios, periódicos regionales, internet, consumo digital de medios) y demandas de información local. Se enfatizó en el consumo de radio, especialmente en las radios locales y en las radios comunitarias con las que trabajamos. Asimismo, se incorporaron aspectos específicos de interés para cada radio que incorporaron algunas intuiciones de sus integrantes sobre determinadas lógicas de consumo: sobre el uso de sus plataformas digitales y redes sociales, sobre el valor y cualidades específicas de las radios reconocidas por la población. El cuestionario contó con unas cuarenta o cuarenta y dos preguntas, según el caso. Para la carga de datos se utilizó Excel y el procesamiento estadístico se realizó con SPSS 22.

Los estudios cualitativos también fueron construidos de manera conjunta. Se trabajó con dos modalidades diferentes. En dos de las ciudades, Alta Gracia y Carlos Paz, se trabajó con entrevistas en profundidad, realizadas en dos encuentros. En cada caso se hicieron quince entrevistas a hombres y mujeres de distintas edades y grupos socioeconómicos. Los criterios para su selección se definieron en función de los resultados de las encuestas realizadas ${ }^{11}$ y se tomaron en consideración a grupos relevantes para los proyectos comunicativos de las radios. En Unquillo y Cosquín se trabajó a través de grupos focales, ya que lo que se buscaba era conocer las líneas argumentales en relación con determinados temas. En la ciudad de Cosquín se realizaron seis grupos focales, en los que el criterio de inclusión para el reclutamiento fue el de trabajar con oyentes de radio entre 31 y 45 años, diferenciados en dos grupos: oyentes de Radio Inédita y no oyentes de esa misma emisora. En Unquillo se realizaron tres grupos focales en los que el criterio de reclutamiento fue la pertenencia a organizaciones de esa ciudad (Lunt y Livingstone, 1999; Jensen y Jankowski, 1993; Jacks, 2011) $)^{12}$.

10 Unquillo cuenta con 19.800 habitantes, Alta Gracia cuenta con 46.858 habitantes, Carlos Paz con 75.315 y Cosquín con 19.458 habitantes según el censo nacional del 2010 que realizó el Instituto Nacional de Estadísticas y Censos.

1 En Alta Gracia se hizo foco en dos grupos: personas de ingresos socioeconómicos medio bajos y jóvenes entre 18 y 30 años, al ser estos los grupos definidos como público-meta de Radio Tortuga. En Carlos Paz se hizo foco en personas de ambos sexos pertenecientes a organizaciones sociales de la ciudad, en función del empeño por profundizar en la interacción con personas organizadas.

12 El número de grupos focales se definió por criterios de factibilidad, aunque en función del análisis también se alcanzó la 
Las dimensiones abordadas en todos los estudios cualitativos fueron modalidades de escucha, valoraciones y sentidos sobre la radio en general y sobre consumo de información. Sin embargo, estas cuestiones se trabajaron siempre en relación con los interrogantes de los integrantes de las radios a través de un proceso por el cual se abordaron las inquietudes a manera de preguntas en clave comunicacional y se construyeron distintas estrategias de investigación. Con dos de esas radios, Tortuga de Alta Gracia e Inédita de Cosquín, se abordaron específicamente los distintos tipos de vínculo que establecen sus audiencias con ellas, el modo en que sus oyentes se configuran como públicos, además de cuestiones informativas, estéticas y de entretenimiento que les permiten a los radioescuchas identificarse con ellas. Por otro lado, se trabajó con oyentes de radio sobre aquellas cuestiones por las cuales no eligen las radios con las que se realizó el estudio.

Otra de las líneas que atravesó los estudios cualitativos estuvo ligada a los sentidos sobre la ciudad que construyen distintos grupos, ya que los integrantes de las radios tenían algunas apreciaciones con respecto a que esas experiencias no estaban siendo nombradas por los medios locales. Esperaban poder dialogar con quienes viven la ciudad cotidianamente, con sus modos de habitarla, nombrarla, moverse, con los conflictos y espacios de intercambio, debate, encuentro y elaboración común. Estas dimensiones se trabajaron en Carlos Paz, Alta Gracia y Unquillo.

En distintas etapas, dos de las radios, Villanos de Carlos Paz y Nativa de Unquillo, plantearon la necesidad de abordar la interacción territorial y comunicacional con las organizaciones locales. Por un lado, porque en el proceso de retroceso y pérdida de derechos como el que estamos viviendo -y que se profundiza en el ámbito comunicacional-, estas radios entienden que una cuestión central para su sustentabilidad es el vínculo con las organizaciones sociales de la comunidad, sea para aportar a esos procesos en términos organizativos, potenciarlas a nivel comunicacional, o para fortalecer su propio proyecto comunicacional. Sus preguntas estaban relacionadas con los modos en que, desde las radios, puedan aportar a los procesos organizativos locales, así como sobre las posibilidades de que esas mismas organizaciones contribuyan a la sustentabilidad de la radio mediante sus contenidos y visibilidad. Para ello, se trabajó en dos relevamientos de organizaciones sociales locales a través de informantes clave. Luego, se sistematizó esa información junto con los contactos de los referentes, de modo tal que sirva como un insumo para la producción informativa. A partir de una muestra construida con las radios, con algunas organizaciones se profundizaron cuestiones vinculadas a su situación organizativa y comunicacional para reconocer el modo en que caracterizan sus posibilidades de expresión pública y sus demandas en ese sentido. Y finalmente, se avanzó en una indagación sobre los consumos mediáticos de los integrantes de esas organizaciones,

saturación. 
sus posibilidades de expresión pública y de reconocimiento sobre conflictos locales, sus abordajes comunicacionales, así como las condiciones y posibilidades para profundizar la interacción comunicativa con las radios.

\section{Resultados}

De este proceso orientado a fortalecer a las emisoras, interesa destacar, en primer lugar, una lógica de investigación en la que se fue trabajando en diálogo permanente con los integrantes de los proyectos radiofónicos. Se recuperaron sus inquietudes, preguntas e intuiciones, no solo para guiar el sentido de las investigaciones realizadas, sino para buscar la manera de abordarlas de manera consistente, teórica y metodológicamente. Y, a partir de los resultados, abrir espacios de encuentro y debate en los que también se fortalezca el diálogo entre espacios académicos y proyectos comunitarios de comunicación (Magallanes et al., 2010; Kejval, 2017).

Los resultados de los estudios cuantitativos permitieron a las radios dimensionar sus niveles de conocimiento y de audiencia. Es destacable que se encontraron niveles importantes de audiencia para las radios comunitarias. En Unquillo, Radio Nativa alcanzó el tercer lugar en audiencia, un 23 \% de la población la escucha. En Alta Gracia y Cosquín, Radio Tortuga y Radio Inédita alcanzan porcentajes aproximados al 10 \% de los oyentes en cada una. En el contexto de crisis de políticas de comunicación que ya reseñamos, este reconocimiento fue un incentivo para fortalecer los colectivos y los proyectos. Además, se convirtió en una herramienta para la búsqueda de alternativas de financiamiento más vinculadas a estrategias publicitarias.

También les permitió reconocer las características particulares de sus oyentes, en algunos casos, un dato relevante fue que sus oyentes no pertenecen al sector socioeconómico ni etario que se habían propuesto interpelar en sus proyectos, lo cual habilitó discusiones en torno a reorientar la propuesta radiofónica o a fortalecer el vínculo con las audiencias actuales. A modo de ejemplo, en Alta Gracia los oyentes de radio Tortuga se concentran en el grupo etario que tiene entre 31 y 45 años, tienen altos niveles educativos y tienen ingresos medios, mientras que el público meta de la radio es de jóvenes de sectores medio bajos. En otros casos, permitió tomar algunas decisiones parar fortalecer y ampliar el vínculo con sectores específicos de oyentes.

Conocer los consumos, prácticas informativas y preferencias de sus oyentes aportó a comprender la trama más amplia de discursos con los que se relacionan y con los que las radios dialogan y disputan ${ }^{13}$. Con esta información, las radios también buscaron fortalecer su propuesta comunicacional, estética e informativa: tanto en Unquillo como en Cosquín, los oyentes de las radios comunitarias con las que trabajamos se

13 En general, los oyentes de las radios muestran un perfil de consumo informativo muy específico en relación a sitios web, canales de TV y radios. 
concentran en el rango de edad entre 31 y 45 años y tienen niveles de ingreso medio y altos niveles educativos. De manera acorde, se tomaron decisiones de producción tendientes a consolidar y ampliar sus audiencias.

Identificar cómo se estructuran las audiencias locales les permitió indagar sobre sus posibilidades de ampliar su audiencia. Por un lado, mediante el análisis de las condiciones para acrecentar el conocimiento de las emisoras en los sectores etarios y sociales que todavía no las conocen: los niveles de desconocimiento de las radios alcanzan el $20 \%$ en todos los casos, y es más alto entre los jóvenes. Además, los estudios permitieron detectar zonas de las ciudades en las que los niveles de desconocimiento son mayores.

También se profundizó el conocimiento sobre quiénes tienen necesidades de información, música o temas y abordajes no satisfechos. Los estudios de audiencia aportaron al reconocimiento de los altos niveles de insatisfacción con la información local y regional (Grillo, 2002). Este es un dato que atraviesa todos los estudios cuantitativos, con niveles de insatisfacción superiores al $60 \%$. Además de una oportunidad de ampliación de las audiencias, fue posible reconocer temáticas de interés sobre las cuales producir información y que se experimentan como zonas de vacancia.

Un resultado destacable de los estudios cuantitativos fue la centralidad del consumo de radios locales que concentran importantes niveles de audiencia luego de las dos radios más escuchadas de la provincia: Cadena 3 y 100.5 (Martínez Luque, 2013). Este dato les permitió redimensionar el valor de las propuestas locales y las expectativas y posibilidades de las radios comunitarias en estos contextos, ya que en todos los casos, las radios locales (comerciales y comunitarias) concentran porcentajes de audiencia que se posiciones desde el tercer al sexto lugar.

De la mano del reconocimiento anterior, el trabajo sobre los distintos sentidos que los oyentes construyen sobre sus ciudades permitió complejizar el modo en que se piensa lo local y buscar alternativas para nombrar y dialogar con las complejas experiencias de vivir cotidianamente en ciudades del Gran Córdoba, y sobre los distintos conflictos que supone el día a día en estas localidades. Son conflictos ambientales, de desarrollo urbano, géneros, infraestructura urbana, transporte, espacios de participación, laborales, dinámicas particulares de exclusión a determinados grupos y sectores sociales que no encuentran un modo de ser representados en los medios locales. Ese reconocimiento plantea el desafío de desarrollar abordajes que permitan visibilizar y articular propuestas.

Los abordajes sobre la interacción comunicativa de las radios con las organizaciones no solo les permitió dimensionar su conocimiento del territorio, también permitió tener indicadores sobre los importantes niveles de reconocimiento que tienen esas organizaciones en relación con la tarea cotidiana de las radios: participación en 
programas, difusión de actividades y conflictos, presencia en los espacios en los que exista la disposición de contar con la radio para desarrollar actividades, en definitiva, ser un ámbito de expresión pública de cada una de las iniciativas. Es destacable cómo las mismas organizaciones propusieron alternativas que colaboren con la ampliación de las audiencias de las radios: generaron propuestas para ampliar el conocimiento de las radios para intervenir en la producción de contenidos sin tener que abandonar sus propias tareas, propusieron contenidos nuevos que consideraban que iban a ampliar la proyección de las radios, ofrecieron sus sedes para transmisiones colaborativas, entre otras. Pero sobre todo, permitió redefinir las preocupaciones de las radios en torno a su incidencia comunicativa (Geertz y Van Oeyen, 2002), ya que ahora quedó planteada desde la necesidad de aportar al trabajo político de esas organizaciones como parte de su propia tarea transformadora. De este modo, se reabrieron las inquietudes por sostener y ampliar sus audiencias.

Finalmente, los estudios en torno a las razones por las cuales un conjunto de oyentes se constituye en audiencia de estas radios, los vínculos que establecen con las emisoras y por qué otros oyentes no adhieren a esa propuesta radiofónica permitieron especificar un conjunto de cualidades específicas en torno a las modalidades de información, la relación con los oyentes, los abordajes sobre la información local y la identidad de las emisoras que constituyeron un aporte para consolidar un conjunto de aspectos que ya venían desarrollando. Pero también para reconocer cuestiones específicas a redefinir: ritmos de conducción, estructuras de programación y musicalización. En definitiva, aportaron elementos para dimensionar la distancia entre su público meta y sus propuestas radiofónicas, lo cual aportó al debate para la consolidación, transformación y ajustes de sus maneras de hacer radio (Martínez Luque, 2012).

\section{Conclusiones y debates}

Creemos que tanto el proceso de investigación como sus resultados posibilitaron reconocimientos que fortalecieron a los medios en cuestión, pero también actualizaron un modo de relación entre investigación y medios comunitarios. En este reencuentro se articularon saberes académicos, inquietudes generadas en la práctica y abordajes teórico metodológicos en los que se produjeron aprendizajes que excedieron a los resultados de investigación. De este proceso surgen un conjunto de saberes e interrogantes en torno al modo en que es posible aportar desde los espacios académicos a consolidar y potenciar estas experiencias de comunicación comunitaria a través del estudio sobre las audiencias del sector comunitario, en función de lo cual sostenemos la necesidad de fortalecer un vínculo que puede ser estratégico, tanto para los medios del sector no lucrativo como para los estudios de comunicación.

Como decíamos antes, estos estudios no solo son relevantes para quienes hacen 
radio, sino que permiten revalorizar la potencialidad del diálogo entre los colectivos radiofónicos y las prácticas de investigación, ya que en muchos casos las inquietudes e intuiciones de quienes sostienen día a día los proyectos constituyen una provocación a la creatividad en los procesos de investigación.

Asimismo, permiten reconocer zonas de vacancia para la producción académica y aportes a cierto sentido común que se instala en el debate sobre las transformaciones en las lógicas de consumo. En particular, permite discutir con ciertas perspectivas vinculadas a la convergencia tecnológica (Jenkins, 2008; 2009; 2010) en las cuales se presenta como un proceso uniforme, lineal, por el cual opera una suerte de desplazamiento de los medios tradicionales hacia los de tipo digital (Orozco y Franco, 2019). Por otro lado, permite discutir con la idea de que ciertos sectores etarios han abandonado los medios de tipo digital (Livingstone, 2002; 2009) para abordar la relación entre consumos de medios de tipo tradicional y digital (Orozco y Franco, 2019), y el modo en que se articulan de manera diferencial en distintos sectores sociales y etarios, así como en contextos semi urbanos de localidades de contextos metropolitanos. Afirmaciones del tipo "los jóvenes no escuchan radio" o "el consumo de radio está cayendo", que a veces se sostienen en datos producidos en grandes ciudades o en percepciones ligadas a la interacción con grupos específicos, pueden ser puestas en cuestión y complejizadas frente a la homogenización que producen estudios nacionales a los que aludimos antes. A modo de ejemplo, en los estudios realizados, los oyentes de radio de las ciudades analizadas concentran porcentajes marcadamente más altos que en los estudios nacionales, producidos solo a partir de datos de grandes centros urbanos: los estudios del Sinca muestran un consumo de radio que concentra alrededor del 70 \% de la población, mientras que en todos nuestros estudios el consumo de este medio está por encima del $80 \%$.

Junto con ello, es posible discutir el consumo de radio de los jóvenes: si bien es el sector etario con menos oyentes de radio, es un sector que presenta muchísimas variaciones en cada una de las ciudades analizadas en cuanto a la cantidad de oyentes que concentra y al consumo de radios locales. Es destacable que en algunas ciudades los oyentes jóvenes no escuchan ninguna radio local, mientras que en otras el consumo de radio se concentra fundamentalmente en las propuestas locales.

De ello, se desprenden interrogantes de tipo académico en torno a las modalidades en las que el proceso de convergencia se articula con las prácticas de consumo audiovisual, mientras que se aporta densidad a la comprensión de las audiencias actuales y sus transformaciones. En términos de la sustentabilidad de los medios comunitarios, abona a las búsquedas en torno a las ofertas disponibles para sectores específicos y con el reconocimiento de sus necesidades, demandas y expectativas. Así, se dibuja una agenda pendiente que requiere continuidad y que podría contribuir 
al conocimiento de los contextos audiovisuales locales.

Un elemento más para destacar es que un aporte sustancial de estos estudios tiene que ver con aportar información para posicionar al sector comunitario en el debate sobre políticas públicas. En nuestro país, el proceso de convergencia ha funcionado como una suerte de excusa para el gobierno de Mauricio Macri para no avanzar con la regulación de los servicios de comunicación audiovisual, ya que se sostiene -en términos generales- que la digitalización de los servicios audiovisuales ampliaría de manera exponencial las posibilidades del sector. Sin embargo, esta lógica de no regulación, junto con resoluciones específicas, solo favoren a la concentración (Monje, Rivero y Zanotti, 2018). Un dato relevante en este sentido tiene que ver con los modos de escucha de radio: los oyentes de radio que usan dispositivos digitales no alcanzan el $10 \%$ en esas localidades. Este dato permite abonar la demanda del sector comunitario de avanzar en el proceso de legalización y regulación del espectro radiofónico, al aplicar lo establecido por la LSCA, incluyendo una reserva del $33 \%$ del espectro para los servicios de comunicación audiovisual sin fines de lucro (Avila Huidrobo y Kejval, 2017).

De este modo, profundizar en este tipo de investigaciones no solo permite aportar a la sustentabilidad de las radios y a provocar nuevos interrogantes para la academia, constituye además un aporte insoslayable a las disputas en torno a políticas públicas de comunicación que fortalezcan al sector comunitario.

\section{Referencias}

Avila Huidrobo, R. y Kejval, L. (2017). Construyendo un mapa de la comunicación junto a las organizaciones populares. Una experiencia de investigación acción participativa. Redes. Revista de Extensión, 39-54.

Córdoba, M. L. y Morales, S. (2018). Conocer para contar. Sobre la experiencia de los estudios sobre Consumos y Audiencias Televisivas del Programa Polos Audiovisuales Tecnológicos. En A. García Vargas, A. Romero, y V. Ligarribay (eds), Sociología(s) y análisis de medios en escala local. Condiciones y situaciones de producción, circulación y reconocimiento en Jujuy y Salta. (pp 17-28). AveSol.

Defensoría del Público (s.f.). ¿En qué cambian a la Ley de Servicios de Comunicación Audiovisual los decretos del Poder Ejecutivo Nacional? Defensoría del Público. https://defensadelpublico. gob.ar/en-que-cambian-a-la-ley-de-servicios-de-comunicacion-audiovisual-los-decretos-delpoder-ejecutivo-nacional/

Doyle, M. (2017). El derecho a la comunicación de los pueblos originarios. Límites y posibilidades de las reivindicaciones indígenas en relación al sistema de medios de comunicación en Argentina (Tesis Doctoral) Facultad de Filosofía y Letras de la UBA, Buenos Aires.

Doyle, M., Meirovich, V. y Morales, S. M. (2019). Informe del primer resultado de Relevamiento de Servicios de Comunicación Audiovisual Comunitarios, Alternativos, Populares, Cooperativos y de Pueblos Originarios. Riccap: Argentina. 
Geertz, A. y Van Oeyen, V. (2002). La radio popular frente al nuevo siglo: estudio de vigencia e incidencia. Signo y pensamiento, 41(21), 122-123.

Giacomini, E., Lois, I. y Coelho, S. (2015). Aproximaciones a la situación de las radios cooperativas de la región AMBA. Perspectivas teórico-metodológicas para el análisis. Revista Idelcoop, (217), 30-41. https://www.idelcoop.org.ar/sites/www.idelcoop.org.ar/files/revista/articulos/pdf/revista217-web-reflexionesydebates-02.pdf

Grillo, M. (2002). Lo local, hoy. Temas y problemas de la comunicación, 9(11), 9-22.

Grillo, M., Papalini, V. y Benítez Larghi, S. (2016). Introducción. En M. Grillo, V. Papalini, y S. Benítez Larghi, Estudios sobre consumos culturales en la Argentina (15-20). Clacso, Codesoc, Pisac.

Grillo, M., y Segura, S. (2016). Consumo y Recepción de Radio y Televisión. En M. Grillo, V. Papalini y S. Benítez (eds.), Estudios sobre consumos culturales en la Argentina Contemporánea (pp. 177- 208). Pisac.

Jacks, N. (2011). Análisis de recepción en América Latina: un recuento con perspectiva a futuro. Ciespal.

Jenkins, H. (2008). Convergence culture. La cultura de la convergencia de los medios de comunicación. Paidós.

Jenkins, H. (2009). Confronting the Challenges of Participatory Culture. MIT Press.

Jenkins, H. (2010). Piratas de textos. Fans, cultura participativa y televisión. Paidós.

Jensen, K. B., y Jankowski, N. W. (eds.) (1993). Metodologías cualitativas de investigación en comunicación de masas. Bosch.

Kejval, L. (2017). Investigar desde la comunicación alternativa, popular y comunitaria. Avatares de la comunicación y la cultura, (13), 31-47. https://publicaciones.sociales.uba.ar/index.php/ avatares/article/view/4871/4004

Kejval, L., y Ávila Huidobro, R. (2017). Los consumos de medios en los territorios, las audiencias de las radios populares y comunitarias. Un relevamiento desde el sur de la ciudad de Buenos Aires. Revista de Ciencias, arte y tecnología, 2, 215-242. https://doi.org/10.35428/cds.v0i2.22

Livingstone, S. (2002). Young people and new media: Childhood and the changing media environment. SAGE.

Livingstone, S. (ed.) (2005a). Audiences and Publics: When Cultural Engagement Matters for the Public Sphere. Intellect Press.

Livingstone, S. (2005b). Critical Debates in Internet Studies: Reflections on an Emerging Field. En J. Curran y M. Gurevitch, (eds.), Mass Media and Society (pp. 9-28). Hodder Arnold.

Livingstone, S. (2009). Children and the Internet: Great Expectations, Challenging Realities. Polity.

Lunt, P., y Livingstone, S. (1999). Rethinking focus groups in media and communications research. Journal of Communication, 46(2), 79-98. https://doi.org/10.1111/j.1460-2466.1996.tb01475.x

Lunt, P. y Livingstone, S. (2010). La audiencia implícita en la formulación de políticas de comunicación: regulación de los medios de comunicación en interés de los ciudadanos y los consumidores. En V. Nightingale (ed), Handbook of Media Audiences (pp. 31 - 49). Blackwell.

Lunt, P. y Livingstone, S. (2015). Is 'mediatization' the new paradigm for our field? A commentary 
on Deacon and Stanyer $(2014,2015)$ and Hepp, Hjarvard and Lundby (2015). Media, Culture and Society, 38(3), 462-470. https://doi.org/10.1177/0163443716631288

Magallanes, C., Ramos, D., Castells, S. y Parra, D. (2010). Un enfoque participativo de investigación para la comunicación comunitaria en México. Folios, (24), 141-162.

Martínez Luque, E. (2012). Movidito, movidito, juntitos todos juntitos. Apuntes sobre las estrategias expresivas de emisoras orientadas a sectores populares de la ciudad de Córdoba. Question, 1(35), 372-386.

Martínez Luque, S. (2013). Trayectorias radiofónicas: aportes para una historia de la radio de Córdoba (Argentina) 1988-2002. Revista Brasileira da História da Mídia, 3(1), 53-63. https:// ri.conicet.gov.ar/bitstream/handle/1 1336/22529/CONICET_Digital_Nro.b8e20dad-2968-451d a381-a5f8c5341b27_A.pdf?sequence $=2$ EisAllowed $=\mathrm{y}$

Mata, M. C. (1997). Medios masivos: lo que nombra el consumo. Revista Estudios, (7-8), 215-222. https://dialnet.unirioja.es/descarga/articulo/5391651.pdf

Mata, M. C. (2012). Desde la experiencia al saber. Sistematización de las experiencias de investigación en audiencias. ALER.

Monje, D. (comp.) (2018). Consumos audiovisuales en la comunidad educativa de la UNVM: caso Uniteve. Ed. UNVM.

Monje, D., y Mercadal, S. (2018). Medios públicos: políticas, actores, estrategias. Eduvim.

Monje, D., y Rivero, E. (2018). Televisión Cooperativa y Comunitaria. Diagnóstico, análisis y estrategias para el sector no lucrativo en el contexto convergente. Convergencia cooperativa.

Monje, D., Rivero, E., y Zanotti, J.M. (2018). Convergencia periférica. Los actores subalternos del mercado info-comunicacional, su importancia y la profundización de condiciones asimétricas. Revista Fibras tecnologías de la comunicación, 21, 60-71. http://papel.revistafibra. info/convergencia-periferica/

Riccap (2019). Informe sobre el relevamiento de Servicios de Comunicación Audiovisual Comunicatarios, Alternativos, Populares, Cooperativos y de Pueblos Originarios en Argentina. Riccap. https://bit. ly/21HPnq8

Orozco, G. (2007). Comunicación social y cambio tecnológico: un escenario de múltiples desordenamientos. En D. De Moraes (coord.), Sociedad mediatizada (pp. 99-117). Gedisa.

Orozco, G., y Miller, T. (2017). La Televisión más allá de sí misma en América Latina. Comunicación y sociedad, (30), 107-127. https://doi.org/10.32870/cys.v0i30.6725

Orozco, G., y Franco, D. (2019) Las audiencias convergentes y su investigación: análisis de recepción transmedial de la serie El Equipo. Derecho a comunicar. Revista científica de la Asociación Mexicana de Derecho a la Información, (5), 46-63. https://www.academia.edu/33141576/ LAS_AUDIENCIAS_CONVERGENTES_Y_SU_INVESTIGACI\%C3\%93N_AN\%C3\%81LISIS_DE_ RECEPCI\%C3\%93N_TRANSMEDIAL_DE_LA_SERIE_EL_EQUIPO

Segura, M. S., Hidalgo, A. L., Kejval, L., Linares, A., Longo, V., Traversaro, N. y Vinelli, N. (2016, diciembre). Los medios comunitarios ante las nuevas políticas de comunicación. Actas 
de Periodismo y Comunicación, 2(1). https://perio.unlp.edu.ar/ojs/index.php/actas/article/ view/4173/3394

Segura, S., Longo, V., Traversaro, N., Linares, A., Vinelli, N. y Espada, A. (2018). Los públicos de medios comunitarios, populares y alternativos en América Latina. El caso argentino. Commons. Revista de Comunicación y Ciudadanía Digital, 7(1), 5-45. https://revistas.uca.es/index.php/cayp/ article/view/4245

Villegas Uribe, E. (2016). Los medios sin fines de Lucro entre la Ley Audiovisual y los decretos. Estrategias, desafíos y debates en el escenario 2009-2015 (M. S. Segura y C. Weckesser) Chasqui. Revista Latinoamericana de Comunicación, 132, 423-426. 